\title{
Usability Comparison Study of Spot-on Formulations of Parasiticides in Cats
}

\author{
Piyarat Chansiripornchai1* and Thunchanok Jantanawaranon2 \\ ${ }^{1}$ Department of Veterinary Pharmacology, Chulalongkorn University, Thailand \\ ${ }^{2}$ Zoetis Limited, Bangkok, Thailand
}

*Corresponding author: Piyarat Chansiripornchai, Associate Professor, Department of Veterinary Pharmacology, Faculty of Veterinary Science, Chulalongkorn University, Thailand.

Received Date: May 06, 2020

Published Date: May 18, 2020

\begin{abstract}
The aim of this study was to evaluate the usability of spot-on parasiticides for cats from the aspects of their stickiness and drying time after application. Eighteen client-owned healthy, munchkin cats (3 males, 15 females), between 9-month-old and 6-year-old, weighing from 1.5-2.9 kg were used in the study. Six spot-on parasiticides which are commercially available in Thailand including selamectin (SE), moxidectin + imidacloprid (MI), praziquantel + emodepside(PE), fipronil + (S)-methoprene + eprinomectin + praziquantel (FMEP), fluralaner (FL) and fipronil + (S)-methoprene (FM) were assessed after their respective application to the skin between shoulder blades of the 18 cats. Stickiness scores (STS) and drying time scores (DTS) were evaluated and recorded at $0.5,1,3,6,9,12,24$ and $48 \mathrm{~h}$ after drug administration (DA). SE and MI groups showed the most satisfying scores of STS at $3 \mathrm{~h}$ after DA, STS in all groups was zero (not sticky) at $48 \mathrm{~h}$ after DA. For DTS, the SE and FL groups had the most satisfying score at 0.5 and $1 \mathrm{~h}$ after DA, DTS in all groups were zero (dry) at $24 \mathrm{~h}$ after DA. Some skin lesions such as erythema and alopecia were found in the MI and FMEP groups but the lesions completely recovered and all cats had normal general health throughout the study. From the study results, all of the 6 spot-on parasiticides are safe for cats and are acceptable for cat owners.
\end{abstract}

Keywords: Cats; Parasiticides; Spot on; Usability

\section{Introduction}

Cats are hosts to a variety of ectoparasites, such as Ctenocephalides felis, Ixodes ricinus and Notoedres cati, and endoparasites, such as Toxocara cati, Ancylostoma tubaeforme and Dypyridium caninum [1]. It is important to treat parasitic infestations to reduce any serious and/or long term health effects on the cat [2]. Moreover, many parasites of cats are zoonotic agents infecting humans from pregnancy (toxoplasmosis), through childhood (Ancylostoma spp. and Toxocara spp.) and the entire life (echinococcosis) [3]. Therefore, proper management practices for the control of parasite infestations are required including the use of efficacious and safe parasiticides [1]. Effective parasiticides, alone or in combination, have been formulated in the last decades for cats [3]. Among the parasiticides available, Spot-on formulations are very popular because of the ease of application in feline patients. Spot-on parasiticides are often well tolerated by cats and hence easier for owners to administer compared to oral medication and to products that must be applied directly to an affected area (such as otic medication for ear mites).

The benefits of a spot-on parasiticides include; a broad spectrum of activity, a low rate of adverse effects and ease of use, increased owner compliance and improved protection of cats against endoparasites and ectoparasites. Fipronil, selamectin, fluralaner, moxidectin,(S)-methoprene, imidacloprid, praziquantel, eprinomectin and emodepside are the well-known commercially available spot-on parasiticides used in cats in Thailand both in the formulation of a single drug and as combination formulation. The quality of each formulation has been evaluated and has shown high efficacy for the treatment and prevention of parasites, such as helminths, mites, fleas and ticks. Thus, there has been a substantial evolution in the convenience of use and spectrum of 
activity of parasiticides available to cat owners. However, there is an ongoing need for formulations with the potential to improve owner compliance with veterinary treatment recommendation [4]. Therefore, the usability of these parasiticides in terms of, such features as satisfaction should be determined in clinical use.

Usability aspects of pharmaceutical preparations are the product characteristics and attributes of a product that enable the patient and/or caregiver in its personal environment and life situation to use the pharmaceutical drug preparation as intended [5]. Unlike drugs that are taken orally, topical drugs are visible to the patient during application, so characteristics like ease of application, stickiness and drying time are important [6]. These parameters are essential since the topical drugs should be non sticky to avoid adherence to the cats' hair-coat and the owners' clothes which may decrease the drug concentration and drug efficacy [7]. Therefore, examining stickiness characteristics, which are associated with the feel, can provide useful information on the clinical use of drug formulation [6].

This study was performed to determine the usability and satisfaction of cat owners with commercial spot -on parasiticides at the recommended dose ranges for cats in Thailand. To our knowledge, this is the first study on the usability of spot-on parasiticides for cats in veterinary clinical practice.

\section{Materials and Methods}

\section{Animals}

Eighteen client-owned healthy, Munchkin cats (3 males, 15 females), between 9 months old and 6 years old, weighing from 1.5$2.9 \mathrm{~kg}$ were used in the study. All the cats were routinely vaccinated against common diseases. During the study no medications were prescribed other than the tested topical arasiticides. Cats were housed in their usual environment and received their usual food and water. Temperature and ventilation were controlled, and the environment was monitored to maintain an ambient temperature of $25-27^{\circ} \mathrm{C}$ and a relative humidity of $55-70 \%$. To be eligible for

Table 1: Categories applied for the assessment of stickiness scores (STS)

\begin{tabular}{|c|c|c|c|}
\hline Score* & $\mathbf{0}$ & $\mathbf{1}$ & $\mathbf{2}$ \\
\hline Criteria & Not sticky & Slightly sticky on the skin & Little tuft of hair \\
\hline
\end{tabular}

*The lowest score indicates the most satisfying result.

Table 2: Categories applied for the assessment of drying time scores (DTS)

\begin{tabular}{|c|c|c|c|c|}
\hline Score* & $\mathbf{0}$ & $\mathbf{1}$ & $\mathbf{2}$ \\
\hline Criterias & Dry & Almost dry & Product remnant persists & Excessive product remnant persist \\
\hline
\end{tabular}

*The lowest score indicates the most satisfying result.

\section{Statistical analysis}

The STS and DTS were analyzed by the Kruskal-Wallis and Mann-Whitney tests (IBM SPSS Statistic 22). A p value of $<0.05$ was considered as statistically significant.

\section{Results}

\section{Stickiness scores (STS)}

The STS in each group of drugs are shown in (Table 3). The results reveal a significant difference $(\mathrm{p}<0.05)$ in the scores among enrollment, cats should not have received any parasiticides within the previous 30 days. Cat owner completed an informed consent form for the inclusion of all cats in a household in the study prior to any enrollment and prior to initiation of treatment. The protocol for animal use and care was approved by the Chulalongkorn University Animal Care and Use Committee, Chulalongkorn University (IACUC number 1931002).

\section{Study design}

A total of 18 household cats were allocated into 6 groups of 3 each according to the completely randomized blocked design on day 0 of the study. Six spot -on parasiticides for cats including; selamectin (SE) (Revolution R, Zoetis, USA), moxidectin + imidacloprid (MI) (Advocate R, Bayer, Germany), praziquantel + emodepside (PE) (Profender R, Bayer, Germany), fipronil+(S)methoprene + eprinomectin + praziquantel (FMEP) (Broadline R, Merial, USA), fluralaner (FL) (Bravecto R, MSD, USA) and fipronil + (S) - methoprene (FM) (Frontline Plus, Merial, USA) were applied to the skin at the midline of the neck between the base of the skull and the shoulder blades of the cats assigned in groups 1 to 6 , respectively. The cats in each group were rotated to receive all 6 drugs in consecutive months except for 3 months in the case of FL with 3 months for the duration of the action. All the procedures for drug application were performed by the owner under the attending veterinarian's instruction.

\section{Assessments}

After the administration of the parasiticides, each cat was observed for $15 \mathrm{~min}$ to determine if any skin irritation was present at the application site. The stickiness score (STS) and drying time score (DTS) of each drug at the administration site were evaluated and recorded by an evaluator based on Tables 1 and 2 at 0.5, 1, 3, $6,9,12,24$ and $48 \mathrm{~h}$ after administration of each drug. Each cat was observed and examined physically by a veterinarian and the cat owner. The general health of each cat was observed twice daily throughout the study. In addition, the cat owner was instructed to observe the cats for any adverse effects (Table 1,2). the drugs at 3, 6, 9, 12 and $24 \mathrm{~h}$ after drug administration (DA). At 3 $\mathrm{h}$ after DA, the lowest STS (1.167) was shown in cats in the SE and MI groups (Figure 1). Interestingly, at 6, 9, 12 and $24 \mathrm{~h}$ after DA, the lowest STS $(0.556,0.278,0.111$ and 0.000 , respectively) were shown in cats in only the SE group. On the other hand, at $12 \mathrm{~h}$ after DA, the STS in cats in the MI group was 0.444 which was lower than that of the PE and FM groups. At $24 \mathrm{~h}$ after DA, zero STS (not sticky) was shown in cats in the SE group while a zero score was shown at $48 \mathrm{~h}$ after DA in other drug groups. 
Table 3: Mean stickiness scores (STS) of 6 spot-on parasiticides at $0.5,1,3,6,9,12,24$ and $48 \mathrm{~h}$ after administration on cats. Different superscripts in each column mean a statistically significant difference $(p<0.05)$.

\begin{tabular}{|c|c|c|c|c|c|c|c|c|}
\hline Drugs & $\mathbf{0 . 5} \mathbf{h}$ & $\mathbf{1} \mathbf{h}$ & $\mathbf{3} \mathbf{h}$ & $\mathbf{6} \mathbf{h}$ & $\mathbf{9} \mathbf{h}$ & $\mathbf{1 2} \mathbf{h}$ & $\mathbf{2 4} \mathbf{h}$ & $\mathbf{4 8} \mathbf{h}$ \\
\hline SE & 2.056 & 1.889 & $1.167^{\mathrm{a}}$ & $0.556^{\mathrm{a}}$ & $0.278^{\mathrm{a}}$ & $0.111^{\mathrm{a}}$ & $0.000^{\mathrm{a}}$ & 0 \\
\hline MI & 2.111 & 2 & $1.167^{\mathrm{a}}$ & $1.389^{\mathrm{b}}$ & $0.833^{\mathrm{a}}$ & $0.444^{\mathrm{a}}$ & $0.056^{\mathrm{a}}$ & 0 \\
\hline PE & 2.056 & 1.944 & $1.833^{\mathrm{a}}$ & $1.556^{\mathrm{b}}$ & $1.222^{\mathrm{b}}$ & $0.889^{\mathrm{a}}$ & $0.444^{\mathrm{b}, \mathrm{c}}$ & 0 \\
\hline FMEP & 2.056 & 2.056 & $2.056^{\mathrm{b}}$ & $1.778^{\mathrm{b}}$ & $1.222^{\mathrm{b}}$ & $0.778^{\mathrm{b}}$ & $0.278^{\mathrm{b}}$ & 0 \\
\hline FL & 1.889 & 1.833 & $1.778^{\mathrm{b}}$ & $1.500^{\mathrm{b}}$ & $1.778^{\mathrm{b}}$ & $0.667^{\mathrm{b}}$ & $0.222^{\mathrm{b}}$ & 0 \\
\hline FM & 2.167 & 2.167 & $2.000^{\mathrm{b}}$ & $1.778^{\mathrm{b}}$ & $1.333^{\mathrm{b}}$ & $0.889^{\mathrm{b}, \mathrm{c}}$ & $0.278^{\mathrm{b}}$ & 0 \\
\hline
\end{tabular}

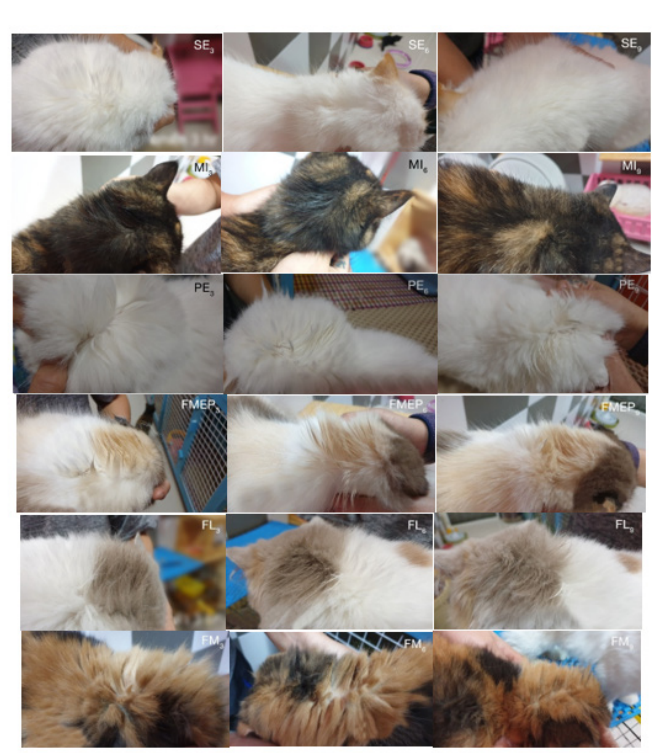

Figure 1: Representative images of stickiness characteristics observed on cats skin.

SE: SE groups; MI: MI groups; PE: PE groups; FMEP: FMEP groups; FL: FL groups and FM: FM groups. Numbers 3, 6 and 9: 3, 6 and 9 h after drug administration.

\section{Drying time scores (DTS)}

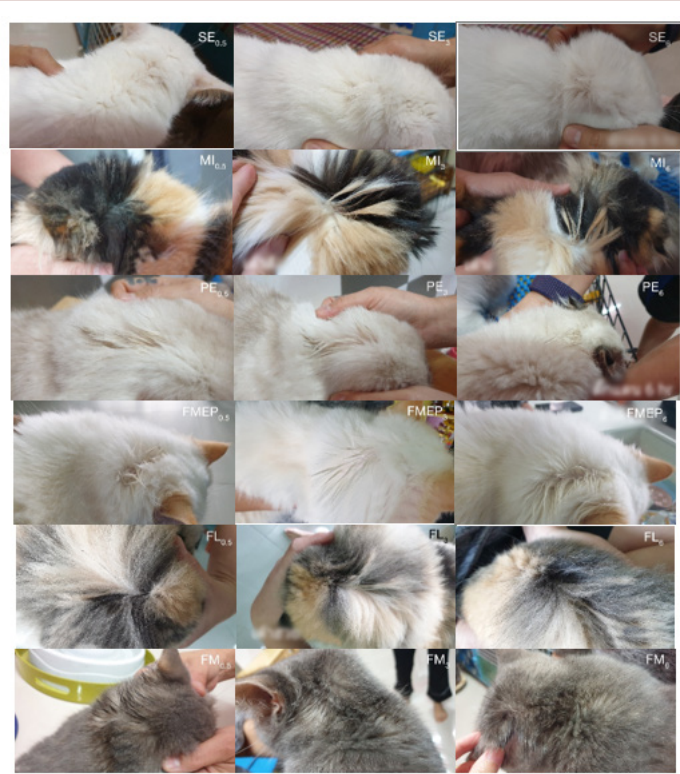

Figure 2: Representative images of drying characteristics observed on cats skin.

SE: SE groups; MI: MI groups; PE: PE groups; FMEP: FMEP groups; FL: FL groups and FM: FM groups. Numbers 0.5, 3 and 6: 0.5, 3 and $6 \mathrm{~h}$ after drug administration. 
Table 4: Mean drying time scores (DTS) of 6 spot-on parasiticides at $0.5,1,3,6,9,12,24$ and $48 \mathrm{~h}$ after administration on cats. Different superscripts in each column mean a statistically significant difference $(p<0.05)$.

\begin{tabular}{|c|c|c|c|c|c|c|c|c|}
\hline Drugs & $0.5 \mathrm{~h}$ & $1 \mathrm{~h}$ & $3 \mathrm{~h}$ & $6 \mathrm{~h}$ & $9 \mathrm{~h}$ & $12 \mathrm{~h}$ & $24 \mathrm{~h}$ & $48 \mathrm{~h}$ \\
\hline SE & $1.500^{\mathrm{a}}$ & $0.889^{\mathrm{a}}$ & $0.167^{\mathrm{a}}$ & $0.167^{\mathrm{a}}$ & 0 & 0 & 0 & 0 \\
\hline MI & $1.833^{\mathrm{b}}$ & $1.556^{\mathrm{b}}$ & $1.167^{\mathrm{a}}$ & $0.500^{\mathrm{a}}$ & 0.167 & 0 & 0 & 0 \\
\hline PE & $1.944^{\mathrm{b}}$ & $1.778^{\mathrm{b}}$ & $1.500^{\mathrm{b}}$ & $0.944^{\mathrm{b}}$ & 0.278 & 0.111 & 0 & 0 \\
\hline FMEP & $1.722^{\mathrm{b}}$ & $1.556^{\mathrm{b}}$ & $1.389^{\mathrm{b}}$ & $0.889^{\mathrm{b}}$ & 0.278 & 0 & 0 & 0 \\
\hline FL & $1.389^{\mathrm{a}}$ & $1.389^{\mathrm{a}}$ & $1.111^{\mathrm{b}}$ & $0.722^{\mathrm{b}}$ & 0.278 & 0.056 & 0 & 0 \\
\hline FM & $1.889^{\mathrm{b}}$ & $1.778^{\mathrm{b}}$ & $1.389^{\mathrm{b}}$ & $0.944^{\mathrm{b}}$ & 0.389 & 0.056 & 0 & 0 \\
\hline
\end{tabular}

The DTS in each group of drugs are shown in Table 4. The results reveal a significant difference $(\mathrm{p}<0.05)$ in the scores among the drugs at $0.5,1,3$ and $6 \mathrm{~h}$ after DA (Figure 2). At 0.5 and $1 \mathrm{~h}$ after DA, the lowest DTS are shown in cats in the SE (1.500) and FL (1.389) groups. At 3 and $6 \mathrm{~h}$ after DA, the lowest DTS (0.167) was shown in cats in the SE group. At $9 \mathrm{~h}$ after DA, the SE group showed a zero score (dry) while the MI and FMEP groups showed zero score at $12 \mathrm{~h}$ after DA. However, there were no significant differences of DTS at 9 and $12 \mathrm{~h}$ after DA in all the drug groups. Moreover, the cats in all of the drug groups showed zero DTS at $24 \mathrm{~h}$ after DA.

\section{Skin lesions after drug administration}

Four cats in the MI group had erythematous skin at the administration site after DA and recovered within 3-9 h. A cat in the FMEP group developed alopecia, red papules and itchy skin within 11 days after DA. In any case, the skin lesions recovered within a month after treatment with a skin supplement. All cats in all the groups of spot-on formulations remained in good general health throughout the study.

\section{Discussion}

In the present study, usability testing in the aspects of stickiness and drying time of spot-on parasiticides for cats were determined. It is well-known that endoparasites and ectoparasites are harmful to the health and welfare of cats therefore, an important part of preventive health care in cats is the control of parasite infestations [4]. Topical spot-on formulation is one of the most popular parasiticides in veterinary practice. The goal of using a topical drug is for local treatment of skin diseases or for transdermal absorption of drugs in the systemic circulation [8]. The spot - on preparations exist in many forms, such as solutions, suspensions and gel which have suitable properties for skin penetration. Frequently used spoton parasiticides in the formulation of single or combination drugs include; fipronil (against ticks and fleas), selamectin (against ticks, fleas, mites and nematodes), fluralaner (against ticks, fleas and mites), moxidectin (against mites and nematodes), (S)-methoprene (against fleas' eggs and larva), imidacloprid (against adult fleas and larva), praziquantel (against cestodes and trematodes), eprinomectin and emodepside (against nematodes) [3]. To facilitate cat owners' compliance, veterinarians should provide evidence to support their decision to select a drug for controlling parasites.

Recently, there have been many reports on the high efficacy of spot-on parasiticides such as SE, MI, PE, FMEP, FL and FM in cats.
Dryden et al. [9] reported an effective eradication of fleas using topical SE and FL in cats. Gracia et al. [10], reported the efficacy of topical FM to control fleas on cats. Tielemans et al. [11], indicated the efficacy of topical FMEP against ticks on cats. Moreover, the efficacy of topical MI and PE for the treatment of nematodes in cats has been revealed $[12,13]$. On the other hand, many reports have revealed a decrease in the efficacy of parasiticides [14]. Possible causes for this decrease in efficacy include reinfection of parasites, climate (sun, rain) and owner failure to comply with treatment instruction or poor compliance [14]. Therefore, usability is an important aspect of concern in treatment application.

Usability is part of the term "user experience" and refers to the ease of use of a product. In order to determine the usability of spoton formulations of antiparasitic drugs, when stickiness and drying time were evaluated. Stickiness and drying time are two of the important properties of spot-on formulations, since stickiness and a greasy feel after application can lead to poor patient compliance. Therefore, there is a need for development of a dosage form which improves the patient compliance [8].

In order to determine the usability of spot-on formulations of parasiticides used in Thailand, stickiness and drying time were evaluated. The results showed the highest quality of both STS and DTS in cats in the SE group (Table 3, 4). At 6, 9, 12 and $24 \mathrm{~h}$ after DA, the cats in the SE group had significantly lower STS than other groups. At $24 \mathrm{~h}$ after DA, zero STS (not sticky skin) was determined in the SE group. However, the cats in the MI, PE, FMEP, FLFM groups had slightly sticky skin at $24 \mathrm{~h}$ after DA, but zero STS in those groups were found at $48 \mathrm{~h}$ after DA (Table 3). Although lower quality of STS was significantly found in the PE group at $24 \mathrm{~h}$ after DA when compared to the other groups, zero STS was also found in this group at $48 \mathrm{~h}$ after DA (Table 3 ). For DTS, the cats in the SE group had zero DTS within $9 \mathrm{~h}$ after DA whereas, the criterion of almost dry was not significantly shown in other groups. However, zero DTS was found at $24 \mathrm{~h}$ after DA in the MI, PE, FMEP, FLFM groups. According to the results, SE performs with good properties of stickiness and drying time for users (cat owners) since the cat owner views a spot-on as having a good feel when it is absorbed well after administration.

The differences in STS and DTS may have been caused by the excipients used in each spot-on formulation, especially the solvents used in each formulation. Although the pharmaceutical preparations of the 6 spot -on formulations in this study are 
solutions, the solvent compositions in each formulation is different. It is well-known that organic solvents are used in pharmaceutical production processes including the drug formulation process [15]. Organic solvents are carbon-based solvents [16]. The main function of solvents is solubilization in liquids and semisolid drugs forms when water cannot be used [15]. Topical formulations usually contain mixed solvent systems to help improves solubility.

The 6 spot-on parasiticides tested in the present study were composed of different solvents. The SE composed of 2 organic solvents include; isopropyl alcohol and dipropylene glycol methyl ether; which have good solvency for a number of substances [17]. In the case of FL which has a high quality of DTS as SE at 0.5 and $1 \mathrm{~h}$ after DA, 2 organic solvents were included; acetone and dimethylacetamide were added. Moreover, glycofurol which is used as a penetration enhancer and solvent in topical and intranasal formulations was also used in the FL. In the case of PE which has a lower quality of STS than other groups at 12 and $24 \mathrm{~h}$ after DA, no solvent was added to this formulation. Our results are in agreement with those previously reported where the solvents form an important component in topical formulation because of helping in the solubilization of the drug and the enhancement of drug permeability $[8,17]$. According to the present results, skin lesions including erythematous skin (22.22\% in MI) and alopecia (5.56 \% in FMEP) were shown even though the lesion was mild and could completely recover. From the results, we can say that all of the spoton formulations tested in this study are safe for cats.

\section{Conclusion}

In the current study, the 6 spot-on parasiticides were not sticky and were completely dry at 48 and $24 \mathrm{~h}$ after DA, respectively. Although there were some significant differences of STS and DTS in the 6 Spot-on parasiticides in this study, the cat owner was still satisfied with all of the formulations. Therefore, we can say that being completely non-sticky and completely dry at 48 and 24 h, respectively after DA was acceptable to the users.

\section{Acknowledgment}

The authors would like to thank associate professor Achara Tawatsin for statistical analysis.

\section{Conflict of Interest}

No conflict of interest.

\section{References}

1. Rehbein S, Capari B, Duscher G, Keidane D, Kirkova Z, et al. (2014) Efficacy against nematode and cestode infections and safety of a novel topical fipronil, (S)-methoprene, eprinomectin and prazuquantel combination product in domestic cats under field conditions in Europe. Vet Parasitol 202(1-2): 10-17.
2. Kvaternick V, Kellermann M, Knaus M, Rehbein S, Rosental J (2014) Pharmacokinetics and metabolism of eprinomectin in cats when administered in a novel topical combination of fipronil, (S)-methoprene, Eprinomectin and praziquantel. Vet Parasitol 202(1-2): 2-9.

3. Otranto D, Little S (2017) Traditional and innovation: selamectin plus sarolaner. A new tool to control endo-and ectoparasites of cats-a European perspective. Vet Parasitol 238: S1-S2.

4. Rohdich N, Zschiesche E, Wolf O, Loehlein W, Pobel T, et al. (2018) Field effectiveness and safety of fluralaner plus moxidectin (Bravecto Plus) against ticks and fleas: a European randomized, blinded, multicenter field study in naturally-infested client-owned cats. Parasit Vectors 11(1): 598.

5. Drumond N, van Riet Nales DA, Karapina Carkit F, Stegeman S (2017) Patients' appropriateness, acceptability, usability and preferences for pharmaceutical preparations: Results from a literature review on clinical evidence. Inter J Pharm 521: 294-305.

6. Inoue Y, Suzuki K, Maeda R, Shimura A, Murata I et al. (2014) Evaluation of formulation properties and skin penetration in the same additive containing formulation. Results Pharm Sci 4: 42-49.

7. Vij NN, Saudagar RB (2014) Formulation, development and evaluation of film - forming gel for prolonged dermal delivery of terbinafine hydrochloride. Int J Pharm Sci Res 5: 537-554.

8. Kathe K, Kathpalia H (2017) Film forming systems for topical and transdermal drug delivery. Asian J Pharm Sci 12: 487-497.

9. Dryden MW, Canfield MA, Bocon C (2018) In-home assessment of either topical fluralaner or Topical selamectin for flea control in naturally infested cats in west central Florida, USA. Parasit Vectors 11.

10. Gracia MJ, Calvete C, Estrada R (2017) Fipronil/(S) methoprene spot-on to control fleas on cats in a field trial in Spain. Pesq Vet Bras 37: 603-607.

11. Tielemans E, Prullage J, Knaus M (2014) Efficacy of a novel topical combination of fipronil, (S)-methoprene, eprinomectin, and praziquantel, against the ticks, Ixodes ricinus and Ixodes scapularis, on cats. Vet Parasitol 202: 59-63.

12. Bohm C, Wolken S, Schnyder M (2015) Efficacy of emodepside/ praziquantel spot-on (Profender) against adult Aelurostrongylus abstrusus nematodes in experimentally infected cats. Parasitol Res 114.

13. Diakou A, Morelli S, Dimzas D (2019) Efficacy of a moxidectin/ imidacloprid spot - on formulation (Advocate) for the treatment of Troglostrongylus brevior in naturally infected cats in a field study in Greece.

14. Fisara P, Guerino F, Sun F (2019) Efficacy of a spot-on combination of fluralaner plus moxidectin (Bravecto Plus) in cats following repeated experimental challenge with a field isolate of Ctenocephalides felis.

15. Grodowska K, Parczewski A (2010) Organic solvents in the pharmaceutical industry. Acta Polo Pharm 67(2): 3-12.

16. Joshi DR, Adhikari N (2019) An overview on common organic solvents and their toxicity. J Pharm Res Inter 28: 1-18.

17. Williams A (2007) Pharmaceutical solvents as a vehicles for topical dosage forms. In: Solvent systems and their selection in pharmaceutics and biopharmaceutics. Biotechnology: Pharmaceutical aspects 6: 403426. 